\title{
Fixed points of terminating mappings in partial metric spaces
}

\author{
Umar Yusuf Batsari®, Poom Kumam® and \\ Sompong Dhompongsa
}

\begin{abstract}
In this paper, we introduce a mapping called a terminating mapping. The existence of a unique, and globally stable fixed point of terminating mappings were established in partial metric spaces. Also, some application theorems in the space of probability density functions and example on quantum operations were presented. The results obtained in this paper, extend, and improve some existing results in the literature.
\end{abstract}

Mathematics Subject Classification. 47H09, 47H10.

Keywords. Terminating mapping, identifying property, regularity property, partial metric, and globally stable fixed point.

\section{Introduction}

The area of fixed point theory received much attention from mathematicians due to its vast scope of applications, see [1,2]. More on applications of fixed point theory can be found in [3-9]. Furthermore, spaces and mappings are very important when studying fixed points; metric spaces, partial metric spaces, fuzzy spaces, smooth spaces, contractive mappings, monotone mappings and so on, see $[1,2,4,10-16]$.

In 1992, Matthews [17] introduced a concept, and basic properties of partial metric (pmetric) functions. The failure of a metric function in computer studies was the primary motivation behind the introductory of the partial metrics[7]. After introducing the partial metric functions, Matthews [7] also proved the partial metric version of the Banach fixed point theorem; this makes the partial metric function relevant in fixed point theory. In 1999, Heckmann [18] established some results using a generalization of the partial metric function called a weak partial metric function. In 2004, Oltra and Valero [19] also generalized the Matthews's fixed point theorem in a complete partial metric space, in the sense of O'Neill. In 2013, Shukla et al. [16] introduced the notion of asymptotically regular mappings in a partial metric space, and established some fixed point results. Recently, Onsod et al. [8] established 
some fixed point results in a complete partial metric space endowed with a graph. Very recently, Batsari and Kumam [20] established the existence, and uniqueness of globally stable fixed points of an asymptotically contractive mappings, using some of the properties of a partial metric function.

Furthermore, another significant area of fixed point theory was brought into light, by the advent of fixed point theorems in ordered spaces, especially the Knaster-Tarski fixed point theorem [9,21]. Later, many important results were established as improvement or generalization of the KnasterTarski fixed point theorem, see [3,5,6,22-24]. In this direction, Heikkila [4] proved some fixed point results of increasing operators in a partially ordered set, and presented some applications in partially ordered Polish spaces. In 2011, Hassen [25] established some fixed point results in ordered partial metric spaces. Some of his results guarantees the existence of a fixed point for non-decreasing mappings. In 2012, Kamihigashi and Stachurski [6,22] established an important criterion for investigating stability of a chain (linear order), in an order theoretic sense. In 2013, Kamihigashi and Stachurski [5] established some fixed points existence, and uniqueness results by using an order-preserving mapping. They complemented their results with some applications on probability distribution functions [5].

In this paper, solely motivated by Kamihigashi et al. [5] and Batsari et al. [20], we extend the work of Kamihigashi et al. [5] from a metric space to a partial metric space. Also, our uniqueness results were obtained with the use of non-contractive mappings. Moreover, application theorems in the space of probability density functions and application example on quantum operations were presented.

\section{Preliminaries}

Let $X$ and $B$ be non empty sets. Let $\mathbb{R}_{+}$denote the set of non-negative real numbers, and $\mathbb{R}$ be the set of real numbers.

Let $\preceq$ be a binary relation on $X$. Then, the relation $\preceq$ is

1. reflexive, if $\forall x \in X, x \preceq x$.

2. antisymmetric, if $x \preceq y$, and $y \preceq x$, imply $x=y \forall x, y \in X$.

3. transitive, if $x \preceq y$, and $y \preceq z$, imply $x \preceq z \forall x, y, z \in X$.

The binary relation $\preceq$ is called a partial order, if it satisfies all the aforementioned conditions $(1-3)[9]$. We call the pair $(X, \preceq)$ a partially ordered set $[9]$.

A partially ordered set $(X, \preceq)$ is called a lattice [9], if for any $a, b \in X$ there exist

1. a least upper bound called "join" or simply $\sup \{a, b\}$ and denoted by $a \cup b[9]$.

2. a greatest lower bound called "meet" or simply inf $\{a, b\}$ and denoted by $a \cap b[9]$.

A lattice $(X, \preceq)$ is complete, if every non-empty subset $\mathrm{A}$ of $\mathrm{X}$ has a least upper bound $\cup A$ and the greatest lower bound $\cap A[9]$.

In view of Kamihigashi et al. [5], a function $\Psi_{b}: X \times X \longrightarrow \mathbb{R}_{+}$for $b \in B \subseteq \mathbb{R}$ is 
1. identifying, if for each $x, y \in X, \Psi_{b}(y, y)=\Psi_{b}(y, x)=\Psi_{b}(x, x), \quad \forall b \in$ $B$, then $x=y$.

2. one dimensional, if $\Psi_{b}$ is independent of $b \in B ; \Psi_{b}=\Psi_{b^{\prime}} \forall b, b^{\prime} \in B$.

3. regular, if for every $x, y, z \in X$ with $x \preceq y \preceq z$, then $\max \left\{\Psi_{b}(x, y)\right.$, $\left.\Psi_{b}(y, z)\right\} \leq \Psi_{b}(x, z)$.

A function $f: X \longrightarrow \mathbb{R}$ with $X \subseteq \mathbb{R}$ is increasing, if $f(x) \leq f(y) \forall x, y \in$ $X$ whenever $x \leq y$.

A sequence $\left\{x_{n}\right\} \subseteq X$ in the space $(X, \preceq)$ is increasing, if $x_{n} \preceq$ $x_{n+1}, \forall n \in \mathbb{N}$.

A partial metric or pmetric on $X[7]$, is a function $p: X \times X \longrightarrow \mathbb{R}_{+}$ such that,

(P1) $\forall x, y \in X, x=y \Longleftrightarrow p(x, x)=p(x, y)=p(y, y)$.

(P2) $\forall x, y \in X, p(x, x) \leq p(x, y)$.

(P3) $\forall x, y \in X, p(x, y)=p(y, x)$.

(P4) $\forall x, y, z \in X, p(x, z) \leq p(x, y)+p(y, z)-p(y, y)$.

Every metric is a partial metric with $p(x, x)=0 \forall x \in X$.

For any partial metric $p$ on $\mathrm{X}$, there exists an induced metric $d_{p}: X \times$ $X \longrightarrow \mathbb{R}_{+}$defined as $d_{p}(x, y)=2 p(x, y)-p(x, x)-p(y, y)$.

Note: If $\Psi_{b}$ satisfy conditions $(\mathrm{P} 1)-(\mathrm{P} 4)$, then it is a partial metric.

Definition 1. If $\Psi_{b}$ is a partial metric, and one dimensional, then we call it a one-dimensional partial metric. If the partial metric $\Psi_{b}$ is not one dimensional then, we call it a partial metric at $b$.

Example 1. Define a mapping $p: X \times X \longrightarrow \mathbb{R}_{+}$by $p(x, y)=\min \{x, y\}$, for $X \subset \mathbb{R}$. Clearly, (P2) fails if $x>y$. Thus, $p$ is not a partial metric.

Example 2. Define a mapping $\Psi_{b}: \mathbb{R} \times \mathbb{R} \longrightarrow \mathbb{R}_{+}$by $\Psi_{b}(x, y)=|b x-b y|$, $b \in B \subset(0,1]$. So, $\Psi_{b}$ is a metric, and a partial metric by implication. But, not one dimensional.

Example 3. Let $b \in B=[-15, \infty)$, define a mapping $\Psi_{b}: F \times F \longrightarrow[0,1]$ by

$$
\Psi_{b}(k, h)=\max \left\{\gamma_{k(b)}, \gamma_{h(b)}\right\},
$$

where $F$ is a family of continuous integrable real-valued functions from $\mathbb{R}$ to $[0,1], \gamma_{k(b)}$ is the infimum of the function $k \in F$ over the interval $[-15, b] \subset B$. So, $\Psi_{b}$ is not a metric. But, it is a partial metric, which is not one dimensional (a partial metric at $b$, for each $b$ ).

Example 4. Let $B \subseteq \mathbb{R}$, define a mapping $\Psi_{b}: \mathbb{R} \times \mathbb{R} \longrightarrow \mathbb{R}_{+}$by

$$
\Psi_{b}\left(x_{1}, x_{2}\right)=\max \left\{x_{1}, x_{2}\right\}
$$

where $x_{1}, x_{2} \in \mathbb{R}$, and $b \in[0,1]$. So, $\Psi_{b}$ is not a metric, but it is a onedimensional partial metric.

An open ball of a partial metric $p: X \times X \longrightarrow \mathbb{R}_{+}$, is a set of the form $B_{\epsilon}^{p}(x):=\{y \in X: p(x, y)<\epsilon\}$ for each $\epsilon>0$, and $x \in X[7]$.

Separation axiom $T_{0}$ [26]: The axiom states that, "for any two distinct points $x, y \in X$, there is an open set $\mathrm{V}$ such that, either $x \in V$ and $y \notin V$ or $y \in V$ and $x \notin V$ ". A topological space fulfilling this axiom is called a $T_{0}$-space. 
Matthews [7] established that every partial metric is a $T_{0}$ topology $\left(\tau_{p}\right)$; $\tau_{p}$ is the topology induced by open balls of the form $B_{\epsilon}^{p}(x)=\{y \in X$ : $p(x, y)<\epsilon\}$ for some $x \in X$.

Every partial metric defines a partial order, using an induced order $\preceq_{p}$ defined as $x \preceq_{p} y \Longleftrightarrow p(x, x)=p(x, y), \forall x, y \in X$.

A sequence $\left\{x_{n}\right\}$ in $(X, p)$ converges with respect to the topology $\tau_{p}$ to a point $x \in X$, if and only if

$$
\lim _{n \longrightarrow \infty} p\left(x_{n}, x\right)=p(x, x)(\text { see }[19]) .
$$

The sequence is Cauchy, if the below limit exists and is finite

$$
\lim _{n, m \longrightarrow \infty} p\left(x_{n}, x_{m}\right)<\infty(\text { see [19]). }
$$

A partial metric space $(X, p)$ is complete, if every Cauchy sequence $\left\{x_{n}\right\}$ in $(X, p)$ converges to a point $x \in X$ such that,

$$
\lim _{n, m \longrightarrow \infty} p\left(x_{n}, x_{m}\right)=p(x, x)(\text { see }[19]) .
$$

Let $(X, p)$ be a partial metric space, and $\left(X, d_{p}\right)$ be the induced metric on $\mathrm{X}$. Then,

1. $\left\{x_{n}\right\}$ is a Cauchy sequence in $(X, p)$, if and only if $\left\{x_{n}\right\}$ is a Cauchy sequence in $\left(X, d_{p}\right)$ (see $\left.[19]\right)$.

2. $(X, p)$ is complete, if and only if $\left(X, d_{p}\right)$ is complete (see [19]).

3. $\lim _{n \longrightarrow \infty} d_{p}\left(x_{n}, x\right)=0 \Longleftrightarrow p(x, x)=\lim _{n \longrightarrow \infty} p\left(x_{n}, x\right)=\lim _{n \longrightarrow \infty}$ $p\left(x_{n}, x_{m}\right)($ see $[19])$.

Definition 2 (see [20]). Let $U: X \longrightarrow X$ be a mapping, and $(X, p, \preceq)$ an ordered partial metric space.

1. Let $x, y \in X$. $U$ is $[x, y]$-order preserving on $X$, whenever $U^{n} x \preceq U^{m} y$ implies $U\left(U^{n} x\right) \preceq U\left(U^{m} y\right), \forall m, n \in\{0,1,2 \cdots\}$.

2. Let $x \in X$. $U$ is $x$-order preserving on $X$, whenever $U^{n} x \preceq U^{m} x$ implies $U\left(U^{n} x\right) \preceq U\left(U^{m} x\right), \forall m, n \in\{0,1,2 \cdots\}$.

Note that, $x$-order preserving is the same as $[x, x]$-order preserving.

3. $U$ is self-order preserving or order preserving on $X$, if we have $y$-order preserving, $\forall y \in X$.

Definition 3. Suppose $X$ is a nonempty set. Suppose $p$ is a real-valued function. Let $\preceq$ be an order define on $X$. A mapping $U: X \longrightarrow X$ is called terminating in an ordered partial metric space $(X, p, \preceq)$, if there exist $t, s \in X$ such that, $U$ is $t$-order preserving, and the following conditions are satisfied:

$$
\begin{aligned}
U^{i} s & \preceq t, \quad \forall i \in \mathbb{N}, \\
t & \preceq U t, \\
p(U t, U t) & \leq p(t, t), \\
p\left(U^{i} x, U^{i} y\right) & \longrightarrow p(U t, U t), \quad \forall x, y \in X .
\end{aligned}
$$

Definition 4. A mapping $U: X \longrightarrow X$ is called semi-terminating in an ordered partial metric space $(X, p, \preceq)$, if there exist $t, s \in X$ such that, $U$ is $t$-order preserving, conditions (2.5) and (2.7) are satisfied. 
Definition 5. Let $U: X \longrightarrow X$ be a terminating mapping in an ordered space $(X, p, \preceq)$. Suppose $t \in X$ is a fixed point of $U$. Then, $t$ is said to be globally stable, if $p\left(t, U^{i} y\right) \longrightarrow p(t, t) \forall y \in X$, as $i \longrightarrow \infty$.

Example 5. Let $F=\left\{g_{i}:[0,10] \longrightarrow \mathbb{R}_{+} \mid g_{i}(x)=x^{2}+10 i, i \in \mathbb{N}\right\}$. Define a mapping $\Psi: F \times F \longrightarrow \mathbb{R}_{+}$by

$$
\Psi\left(g_{i}, g_{j}\right)=\max \left\{A_{i}, A_{j}\right\}
$$

where $A_{i}$ is the area of the region bounded by both $g_{i} \in F$ and the horizontal axis. Also, define a mapping $U: F \longrightarrow F$ by

$$
U\left(g_{i}\right)=g_{i-1},
$$

where $g_{i-1}$ is the nearest function (curve/graph) below $g_{i} \in F$. Define the order relation $\preceq$ on $F$ by $g_{i} \preceq g_{j} \Longrightarrow A_{j} \leq A_{i}$. Consider (2.8), (2.9), $t=$ $g_{1}$, and $s=g_{8}$. Conditions (2.4)-(2.7) are satisfied (see Graph of $g_{1}-g_{8}$ ). Thus, $U$ is a terminating mapping.

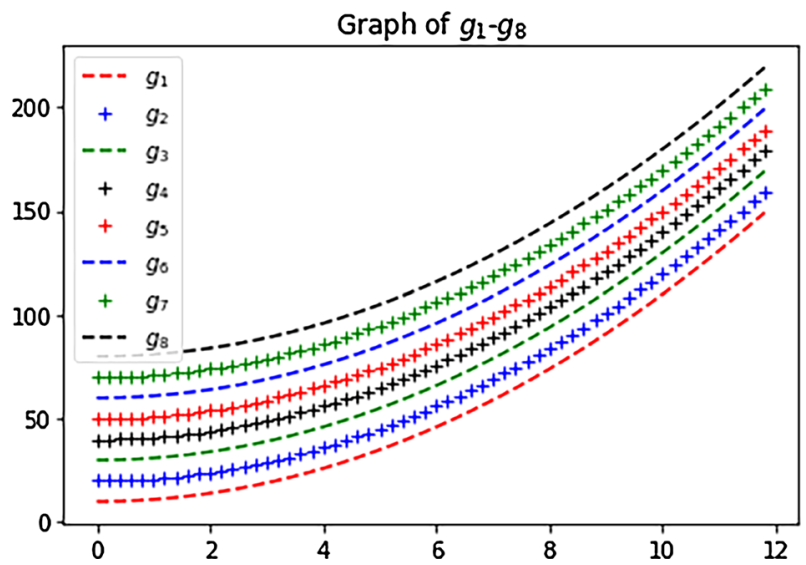

Let $\mathfrak{P}_{S}$ be the space of all probability measures on $(S, \mathfrak{B})$, where $\mathrm{S}$ is a topological space equipped with Borel sets $\mathfrak{B}$. A sequence $\left\{\mu_{n}\right\} \subset \mathfrak{P}_{S}$ is said to be tight, if for all $\epsilon>0$, there exists a compact set $K \subset S$ such that, $\mu_{n}(S \backslash K) \leq \epsilon$ for all $n[6]$.

Note that, we denote $U^{0}$ to be the identity mapping throughout this paper.

Let $\mathcal{B}(H)$ be the set of bounded linear operators on Hilbert space $H$ and $\mathcal{A}=\left\{A_{i}, A_{i}^{\dagger}: i=1,2,3 \cdots\right\}$ be a set of operators where $A_{i} \in \mathcal{B}(H)$ satisfy $\sum A_{i}^{\dagger} A_{i} \leq I$, where $A_{i}^{\dagger}$ is the conjugate transpose of $A_{i}$.

A map $\mathcal{E}: \mathcal{B}(H) \longrightarrow \mathcal{B}(H)$ of the form $\mathcal{E}_{\mathcal{A}}(B)=\sum A_{i} B A_{i}^{\dagger}$ is called a quantum operation.

\section{Main results}

From what will follow in this section, we consider $X$ to be nonempty, $\preceq$ as an order defined on $\mathrm{X}$, and $p: X \times X \longrightarrow \mathbb{R}_{+}$a well-defined function. Suppose $U$ be a self-mapping on $X$. We assume the followings: 
Assumption 3.1. $\preceq$ is transitive.

Assumption 3.2. $p$ is identifying.

Assumption 3.3. $p$ is regular.

Assumption 3.4. If $x \in X$ is a fixed point of $U$, then $p(y, x)=p(x, y) \forall y \in X$.

Theorem 3.1. Suppose $\preceq$ is reflexive. $U$ has a fixed point, iff there exist $s, t \in X$ such that, $U$ is t-order preserving, and the following conditions are satisfied:

$$
\begin{aligned}
p\left(U^{i} s, U^{i} t\right) & \longrightarrow p(U t, U t), \\
U^{i} s & \preceq t, \quad \forall i \in \mathbb{N}, \\
t & \preceq U t, \\
p(U t, U t) & \leq p(t, t) .
\end{aligned}
$$

Proof. Let $x \in X$ be a fixed point of $U$. Consider $x=t=s$, for $\preceq$ being reflexive, then $U$ is both $x$-order preserving, and $[x, x]$-order preserving. As a consequence, we deduce

$$
p\left(U^{i} x, U^{i} x\right)=p(x, x)=p(U x, U x) .
$$

From (3.5), we have (3.4), and $p\left(U^{i} x, U^{i} x\right) \longrightarrow p(U x, U x)$. Hence, (3.1) follows immediately. Since $\preceq$ is reflexive, and $x$ is a fixed point, then $x \preceq U x$, and $U^{i} x \preceq x \forall i \in \mathbb{N}$. Therefore, (3.2) and (3.3) follow.

Conversely, suppose (3.1)-(3.4) hold. For $\preceq$ reflexive, and the mapping $U$ being $t$-order preserving on $X$, we have $U t \preceq U t \preceq U^{i} t$. Using Assumption 3.1, Assumption 3.3, (3.2), and (3.3), we deduce that,

$$
U^{i} s \preceq t \preceq U^{i} t \text {. }
$$

By regularity of $p$, and (3.4), we proceed as

$$
\begin{aligned}
p(U t, U t) & \leq p(t, t) \\
& \leq p(t, U t) \\
& \leq p\left(t, U^{i} t\right) \\
& \leq p\left(U^{i} s, U^{i} t\right) \\
& \longrightarrow p(U t, U t) .
\end{aligned}
$$

So, the above inequalities imply $p(U t, U t)=p(t, U t)=p(t, t)$. As a consequence of the identifying property of $p$, we have $t=U t$. Hence, $t$ is a fixed point of $U$.

Example 6. Consider the collection $\mathcal{M}$ of $2 \times 2$ matrices with real entries. Define a mapping $U: \mathcal{M} \longrightarrow \mathcal{M}$ by

$$
U(A)=M A M^{\dagger}, M=\left[\begin{array}{ll}
0 & 1 \\
1 & 0
\end{array}\right] .
$$

Define an order relation $\preceq$ on $\mathcal{M}$ by $A \preceq B$ iff $a_{i j} \geq b_{i j}$ for $A, B \in \mathcal{M}$ and $i, j \in\{1,2\}$. For a functional $p: \mathcal{M} \times \mathcal{M} \longrightarrow \mathbb{R}_{+}$, define

$$
p(A, B)=\left|a_{11}-b_{11}+a_{12}-b_{12}+a_{21}-b_{21}+a_{22}-b_{22}\right| .
$$


So, it is easy to check that all conditions and Assumptions of the above Theorem 3.1 are satisfied. Therefore, fixed points set of $U$ denoted by $F(U)$ is

$$
F(U)=\left\{\left[\begin{array}{ll}
a & b \\
b & a
\end{array}\right], a, b \in \mathbb{R}\right\} .
$$

Corollary 3.2. Suppose $(X, p)$ is a partial metric space associated with a partial order $\preceq$. Let $U$ be a self- and order-preserving mapping on $X$. Then, $U$ has a fixed point, iff there exist $s, t \in X$ such that (3.1)-(3.4) are satisfied.

Lemma 3.3. Suppose $\preceq$ is reflexive. Let $p$ be a partial metric on $X$. If $U$ is a terminating mapping on $X$, then $U$ has a unique globally stable fixed point.

Proof. Assume there exist $s, k \in X$ such that $U$ is a terminating mapping, and $k$-order preserving. Then, $k$ and $s$ satisfy (2.4)-(2.6). In view of Theorem $3.1, \mathrm{k}$ is a fixed point of $U$.

We next verify global stability of $k$ as follows: Let $y \in X$ then,

$$
\begin{aligned}
p\left(k, U^{i} y\right) & =p\left(U^{i} k, U^{i} y\right) \\
& \longrightarrow p(U k, U k) \\
& =p(k, k) .
\end{aligned}
$$

Therefore, $k$ is a globally stable fixed point of $U$.

Moreover, suppose $U$ have two fixed points say $h$ and $k$. Then, we have

$$
\begin{aligned}
p(h, k) & =p\left(h, U^{i} k\right) \\
& \longrightarrow p(h, h) \\
& =p(h, h) .
\end{aligned}
$$

Hence, $p(h, k)=p(h, h)$.

Similarly, we have $p(h, k)=p(k, k)$. Thus, $p(h, h)=p(h, k)=p(k, k)$. Using identifying property of $p$, we have $h=k$.

Theorem 3.4. Let $\preceq$ be reflexive. Suppose the function p satisfies conditions $(\mathrm{P} 2)$ and $(\mathrm{P} 4)$. Then, $U$ has a unique globally stable fixed point, iff $U$ is a terminating mapping.

Proof. In view of Theorem 3.1, and Lemma 3.3, it suffices only to show that, if $p$ satisfies (P4), and $U$ has a globally stable fixed point, then $U$ is a terminating mapping. Let $x, y, t \in X$ such that, $t$ is a globally stable fixed point of $U$. From Theorem 3.1, $t$ satisfies (2.4)-(2.6) for some $s \in X$. Furthermore

$$
\begin{aligned}
p\left(U^{i} x, U^{i} y\right) & \leq p\left(U^{i} x, t\right)+p\left(t, U^{i} y\right)-p(t, t) \\
& \longrightarrow p(t, t)+p(t, t)-p(t, t) \\
& =p(t, t) \\
& =p(U t, U t) .
\end{aligned}
$$

Therefore, $U$ is a terminating mapping. 


\section{Complete space case}

In addition to Assumptions 3.1-3.4, we add the following assumptions:

Assumption 4.1. $(X, p)$ is a complete partial metric space, and $\preceq$ is a reflexive order defined on $X$.

Assumption 4.2. For any increasing sequence $\left\{x_{i}\right\}_{i \in \mathbb{N}} \subset X$ converging to $x \in X$, we have $x_{i} \preceq x \forall i \in \mathbb{N}$.

Assumption 4.3. Let $\left\{x_{i}\right\}_{i \in \mathbb{N}} \subset X$ be any increasing sequence that converges to $x \in X$. If there exists $y \in X$ such that, $x_{i} \preceq y \forall i \in \mathbb{N}$, then $x \preceq y$.

Assumption 4.4. Define $\left\{x_{i}\right\}$, and $\left\{y_{i}\right\}$ on $X$ by $x_{i}=U^{i} x_{0}$, and $y_{i}=U^{i} y_{0}$ respectively. Let $\left\{x_{i}\right\}$ be increasing and convergent. Let $\left\{y_{i}\right\}$ be convergent. Suppose exist $h \in X$ such that, $x_{i} \preceq h \preceq y_{i}$, and $\lim _{i \longrightarrow \infty} p\left(x_{i}, h\right)=$ $\lim _{i \longrightarrow \infty} p\left(y_{i}, h\right)=p(h, h)$. Then, $x_{i} \preceq h \preceq U h \preceq y_{i} \forall i \in \mathbb{N}$, and $p(h, h) \geq p(U h, U h)$.

Theorem 4.1. Suppose there exists a positive natural number $\beta$ such that, for any $x, y \in X$, we have

$$
x \preceq y \Longrightarrow p\left(U^{i} x, U^{i} y\right) \longrightarrow \beta .
$$

Suppose there exist $t, s \in X$ such that $U$ is $t$-order preserving on $X,[s, t]$ order preserving on $X$, and

$$
\begin{aligned}
t & \preceq U t, \\
U^{i} t & \preceq s, \quad \forall i \in \mathbb{N} .
\end{aligned}
$$

Then, U has a fixed point.

Proof. For a fixed point to exists, it is enough to show the existence of $\hat{x}, t$ satisfying conditions (3.1)-(3.4).

Let $x_{i}=U^{i} t \forall i \in \mathbb{N}$. It follows from (4.2), and $t$-order preserving condition on $U$ that, $\left\{x_{i}\right\}_{i \in \mathbb{N}}$ is increasing. We next need to show the sequence $\left\{x_{i}\right\}$ is Cauchy, using (4.1)-(4.3), and regularity of $p$. Let $\epsilon>0$, from (4.1)(4.3) there exists $m \in \mathbb{N}$ such that, $\left|p\left(U^{m} t, U^{m} s\right)-\beta\right|<\epsilon$. Let $j, k \in \mathbb{N}$, suppose $k>j>m$, and $N=k-m$. Clearly, $x_{m} \preceq x_{j} \preceq x_{k}$, from which we proceed as

$$
\begin{aligned}
\left|p\left(x_{j}, x_{k}\right)-\beta\right| & \leq\left|p\left(x_{m}, x_{k}\right)-\beta\right| \\
& =\left|p\left(U^{m} t, U^{k} t\right)-\beta\right| \\
& =\left|p\left(U^{m} t, U^{m} U^{N} t\right)-\beta\right| \\
& \leq\left|p\left(U^{m} t, U^{m} s\right)-\beta\right|<\epsilon .
\end{aligned}
$$

Hence, $\lim _{j, k \longrightarrow \infty} p\left(x_{j}, x_{k}\right)=\beta$. So, $\left\{x_{i}\right\}_{i \in \mathbb{N}}$ is Cauchy. By completeness of $(X, p)$, there exists $\hat{x} \in X$ such that, $x_{i} \longrightarrow \hat{x}$, i.e., $\lim _{n \longrightarrow \infty} p\left(x_{i}, \hat{x}\right)=$ $p(\hat{x}, \hat{x})$. Thus, (3.2) follows immediately from Assumption 4.2.

Furthermore, using Assumption 4.2, t-order preserving property of $U$, and (4.2), we have

$$
t \preceq U^{i} t \preceq \hat{x} \quad \forall i \in \mathbb{N} .
$$


Using $t$ and $\hat{x}$ in (4.1), and (4.4), we have $p\left(U^{i} t, U^{i} \hat{x}\right) \longrightarrow \beta$. Also, from Assumption 4.3, (4.3), and (4.4), we have

$$
t \preceq x_{i} \preceq \hat{x} \preceq s \quad \forall i \in \mathbb{N} .
$$

From (4.5), Assumption 4.3, and for $U[t, s]$-order preserving, we have

$$
x_{i} \preceq \hat{x} \preceq U^{i} s \quad \forall i \in \mathbb{N} .
$$

Considering (4.1), (4.5), (4.6), and regularity of $p$, we have

$$
\begin{aligned}
p(\hat{x}, \hat{x}) & \leq p\left(x_{i}, \hat{x}\right) \\
& \leq p\left(x_{i}, U^{i} s\right) \\
& =p\left(U^{i} t, U^{i} s\right) \\
& \longrightarrow \beta .
\end{aligned}
$$

Thus, from above inequalities, we have $p(\hat{x}, \hat{x}) \leq \beta$. To show the other way round, we proceed as follows.

$$
\begin{aligned}
p\left(x_{m}, x_{n}\right) & \leq p\left(x_{m}, \hat{x}\right)+p\left(\hat{x}, x_{n}\right)-p(\hat{x}, \hat{x}) \\
& \longrightarrow p(\hat{x}, \hat{x})+p(\hat{x}, \hat{x})-p(\hat{x}, \hat{x}) \\
& =p(\hat{x}, \hat{x}) .
\end{aligned}
$$

Hence, from above inequalities, we deduce that $\beta \leq p(\hat{x}, \hat{x})$. Therefore, $\beta=$ $p(\hat{x}, \hat{x})$. From $(4.6)$, and regularity of $p$, we have

$$
\begin{aligned}
p(\hat{x}, \hat{x}) & \leq p\left(U^{i} t, \hat{x}\right) \\
& \leq p\left(U^{i} t, U^{i} s\right) \\
& \longrightarrow p(\hat{x}, \hat{x}) .
\end{aligned}
$$

Therefore, $\lim _{i \longrightarrow \infty} p\left(U^{i} s, \hat{x}\right)=p(\hat{x}, \hat{x})$. Using $x_{i}=U^{i} t, y_{i}=U^{i} s$, (4.6), $p\left(U^{i} s, \hat{x}\right)=p(\hat{x}, \hat{x})$, and Assumption 4.4 we have $x_{i} \preceq \hat{x} \preceq U \hat{x} \preceq y_{i} \forall i \in \mathbb{N}$, and $p(U \hat{x}, U \hat{x}) \leq p(\hat{x}, \hat{x})$. Hence, (3.1), (3.3), and (3.4) immediately follow. So, from the prove of Theorem $3.1, \hat{x}$ is a fixed point of $U$.

\subsection{Some applications to probability density functions}

Let $\mathfrak{F}$ be the set of all probability density functions on $\mathbb{R}$. Let $U$ be a selfmapping on $\mathfrak{F}$.

Define $p: \mathfrak{F} \times \mathfrak{F} \longrightarrow \mathbb{R}_{+}$as follows:

$$
p(f, g)=\underline{A_{f}, g}+\underline{\underline{A_{f}, g}}
$$

where $\underline{A_{f}, g}$ is the area of the region under both $f$ and $g$ while $\underline{A_{f}, g}$ is the area of the region between $\mathrm{f}$ and $\mathrm{g}$.

Claim: The function $p$ defined by (4.7) is a partial metric.

Proof. Let us denote the area under the density functions $f, g$, and $h$ by $A(f), A(g)$, and $A(h)$, respectively. Also, let the alphabets a, b, c, d, e, and $\mathrm{k}$ represent the area of regions between or under the density functions. Below are the eight basic possible permutations(arrangements) that we can have with the density functions $f, g$ and $h$. To show $p$ is a partial metric, we need to check the conditions (P1), (P2), and (P3) for both Figures 1 and 2; two cases only. While, (P4) is verified using Figs. 3, 4, 5, 6, 7 and 8. 
1. To check for condition (P1), we have the followings from Fig. 1. $p(f, g)=a+b+c=1+c$ or $p(f, g)=a+b+c=a+1$. Also, $p(f, f)=a+b=1$ and $p(g, g)=b+c=1$. So, if $p(f, g)=p(f, f)=$ $p(g, g)$, then $p(f, g)=b=1$. In each case, it implies $a=0$, and $c=0$. Thus, $f=g$.

Conversely, if $f=g$, then $1=p(f, f)=p(f, g)=p(g, g)$. Therefore, condition (P1) holds in Fig. 1.

2. To check for condition (P2) in Fig. 1, we proceed as follows: $p(f, f)=a+b=1, p(g, g)=b+c=1$, and $p(f, g)=a+b+c=1+a$ or $p(f, g)=a+b+c=1+c$. Thus, $p(f, f)=1 \leq 1+c=p(f, g)$. Also, $p(g, g)=1 \leq 1+a=p(f, g)$. Hence, condition (P2) holds in Fig. 1.

3. To check for condition (P3) in Fig. 1, observe that, $p(f, g)=a+b+c=$ $c+b+a=p(g, f)$. So, condition (P3) holds in Fig. 1 . follows:

Similarly, to check for conditions (P1)-(P3) in Fig. 2, we proceed as

1. Observe that,

$$
p(f, g)=a+b=2 \text {. Also, } p(f, f)=a=1 \text {, and } p(g, g)=b=1 \text {. So, }
$$
if $p(f, g)=p(f, f)=p(g, g)$, then $p(f, g)=a+b=1$. Which means, either $a=0$ or $b=0$. In any case, $f=g$.

Conversely, if $f=g$, then $p(f, g)=p(f, f)=p(g, g)=1$. Thus, condition (P1) holds in Fig. 2.

2. To check for condition (P2) in Fig. 2, observe that, $p(f, f)=a=$ $1, p(g, g)=b=1$, and $p(f, g)=a+b=2$. So we have, $p(f, f)=$ $1<2=p(f, g)$, and $p(g, g)=1<2=p(f, g)$. Therefore, condition (P2) holds in Fig. 2.

3. To check for condition (P3) in Fig. 2, it is clear that, $p(f, g)=a+b=$ $b+a=p(g, f)$. Thus, condition (P3) holds in Fig. 2.

In view of the above facts, conditions (P1)-(P3) hold for the function $p(f, g)=\underline{A_{f}, g}+\underline{A_{f}, g}$. Next, we show that, condition (P4) hold in all the remaining six figures (Figs. 3, 4, 5, 6, 7, 8).

Specifically, we are to show the condition $p(f, g) \leq p(f, h)+p(h, g)-$ $p(h, h)$ is true for all the possible permutations of $f, g$, and $h$ across the six figures (Figs. 1, 2, 3, 4, 5, 6, 7).

Using Fig. 3, we have $p(f, g)=a+b, p(f, h)=a+c, p(h, g)=b+c$, and $p(h, h)=c$. Consequently,

$$
\begin{aligned}
p(f, g) & =a+b \\
& <a+b+c \\
& =(a+c)+(b+c)-c \\
& =p(f, h)+p(h, g)-p(h, h) .
\end{aligned}
$$

Hence, $p(f, g) \leq p(f, h)+p(h, g)-p(h, h)$ in Fig. 3 .

Using Fig. 4, we have $p(f, g)=a+b+d+e, p(f, h)=b+c+d+$ $e, p(h, g)=a+b+c+d$, and $p(h, h)=b+c+d$. So that, 


$$
\begin{aligned}
p(f, g) & =a+b+d+e \\
& <a+b+c+d+e \\
& =(b+c+d+e)+(a+b+c+d)-(b+c+d) \\
& =p(f, h)+p(h, g)-p(h, h) .
\end{aligned}
$$

Thus, $p(f, g) \leq p(f, h)+p(h, g)-p(h, h)$ in Fig. 4 .

Using Fig. 5, we have $p(f, g)=a+b+c, p(f, h)=a+c+d, p(h, g)=$ $b+c+d$, and $p(h, h)=c+d$. So that,

$$
\begin{aligned}
p(f, g) & =a+b+c \\
& <a+b+c+d \\
& =(a+c+d)+(b+c+d)-(c+d) \\
& =p(f, h)+p(h, g)-p(h, h) .
\end{aligned}
$$

Thus, $p(f, g) \leq p(f, h)+p(h, g)-p(h, h)$ in Fig. 5 .

Using Fig. 6, we have $p(f, g)=a+b+c, p(f, h)=a+b+d, p(h, g)=$ $b+c+d$, and $p(h, h)=d$. Consequently,

$$
\begin{aligned}
p(f, g) & =a+b+c \\
& <a+2 b+c+d \\
& =(a+b+d)+(b+c+d)-d \\
& =p(f, h)+p(h, g)-p(h, h) .
\end{aligned}
$$

Thus, $p(f, g) \leq p(f, h)+p(h, g)-p(h, h)$ in Fig. 6 .

Using Fig. 7, we have $p(f, g)=a+b+c+e+k, p(f, h)=a+b+c+$ $d+e, p(h, g)=b+c+d+e+k$, and $p(h, h)=b+c+d+e$. So that,

$$
\begin{aligned}
p(f, g) & =a+b+c+e+k \\
& <a+b+c+d+e+k \\
& =(a+b+c+d+e)+(b+c+d+e+k)-(b+c+d+e) \\
& =p(f, h)+p(h, g)-p(h, h) .
\end{aligned}
$$

Thus, $p(f, g) \leq p(f, h)+p(h, g)-p(h, h)$ in Fig. 7 .

Using Fig. 8, we have $p(f, g)=a+b+c+d, p(f, h)=a+b+d+$ $e, p(h, g)=b+c+d+e$, and $p(h, h)=d+e$. So

$$
\begin{aligned}
p(f, g) & =a+b+c+d \\
& <a+2 b+c+d+e \\
& =(a+b+d+e)+(b+c+d+e)-(d+e) \\
& =p(f, h)+p(h, g)-p(h, h) .
\end{aligned}
$$

Thus, $p(f, g) \leq p(f, h)+p(h, g)-p(h, h)$ in Fig. 8. Therefore, the function $p$ defined by (4.7) is indeed a partial metric function.

Now, define an order $\preceq_{p}$ on $\mathfrak{F}$ by

$$
f \preceq_{p} g \Longleftrightarrow p(f, g) \leq p(f, f) .
$$

The order $\preceq_{p}$ is a partial order. 


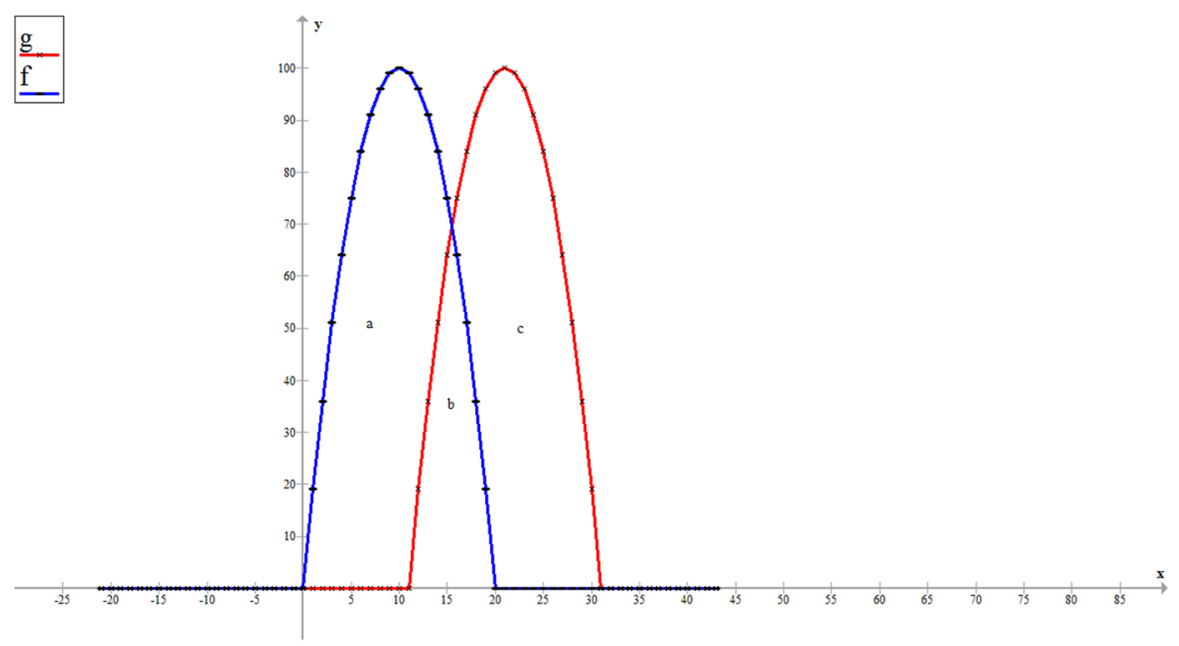

Figure 1. $A(f) \bigcap A(g) \neq \emptyset$

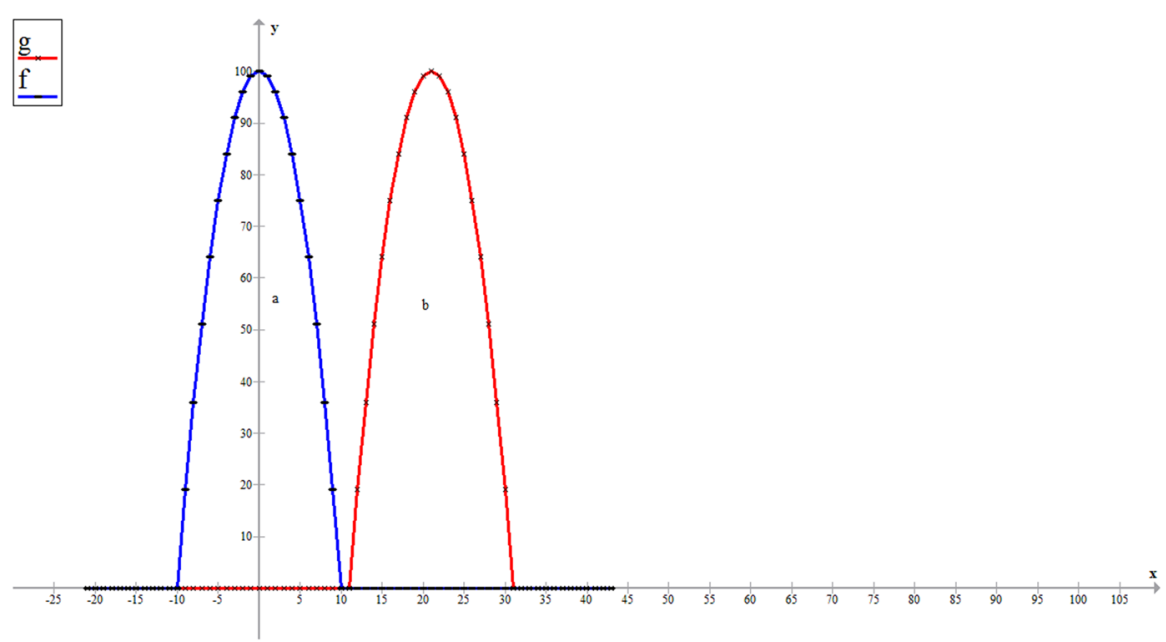

Figure 2. $A(f) \bigcap A(g)=\emptyset$

It is easy to verify that, under the definitions of $\preceq_{p}$ (4.8), and $p$ (4.7) above, Assumptions 3.2-3.4 are satisfied. The application theorems are as follows:

Theorem 4.2. The self-mapping $U$ has a unique globally stable invariant probability density function, iff $U$ is a terminating mapping on $\mathfrak{F}$.

Proof. The prove follows immediately from Theorem 3.4 and Lemma 3.3.

Theorem 4.3. Suppose $(\mathfrak{F}, p)$ is a complete partial metric space. The mapping $U$ has a unique globally stable invariant probability density function, iff $U$ is 


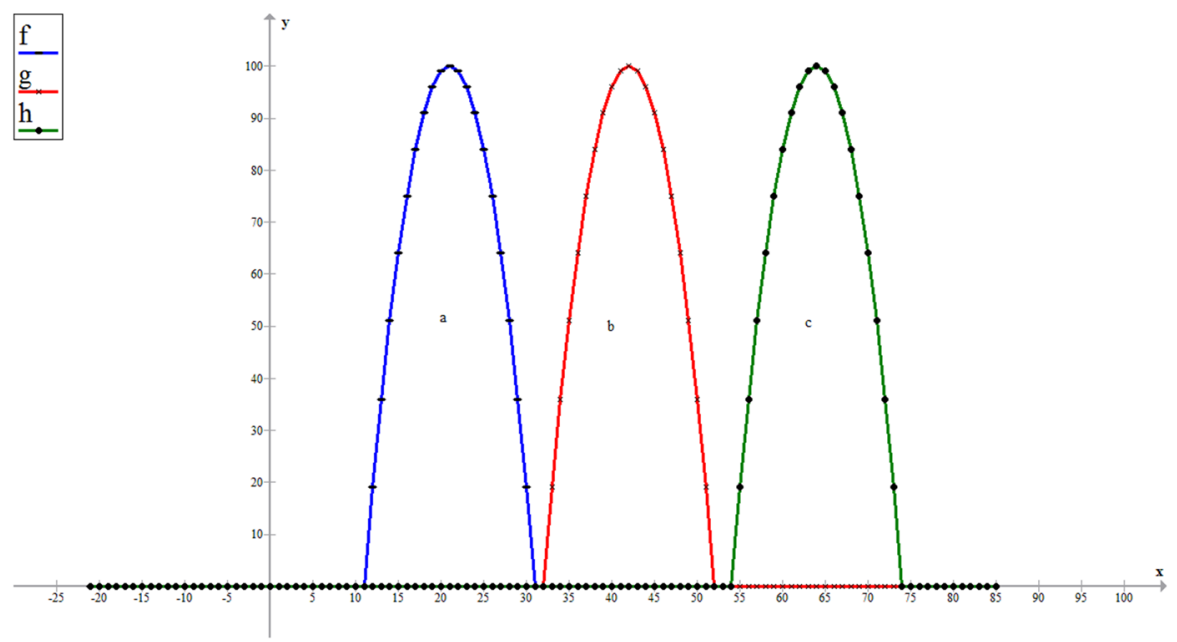

Figure 3. $A(f) \bigcap A(h)=\emptyset, A(g) \bigcap A(h)=\emptyset$ and $A(f) \bigcap A(g)=\emptyset$

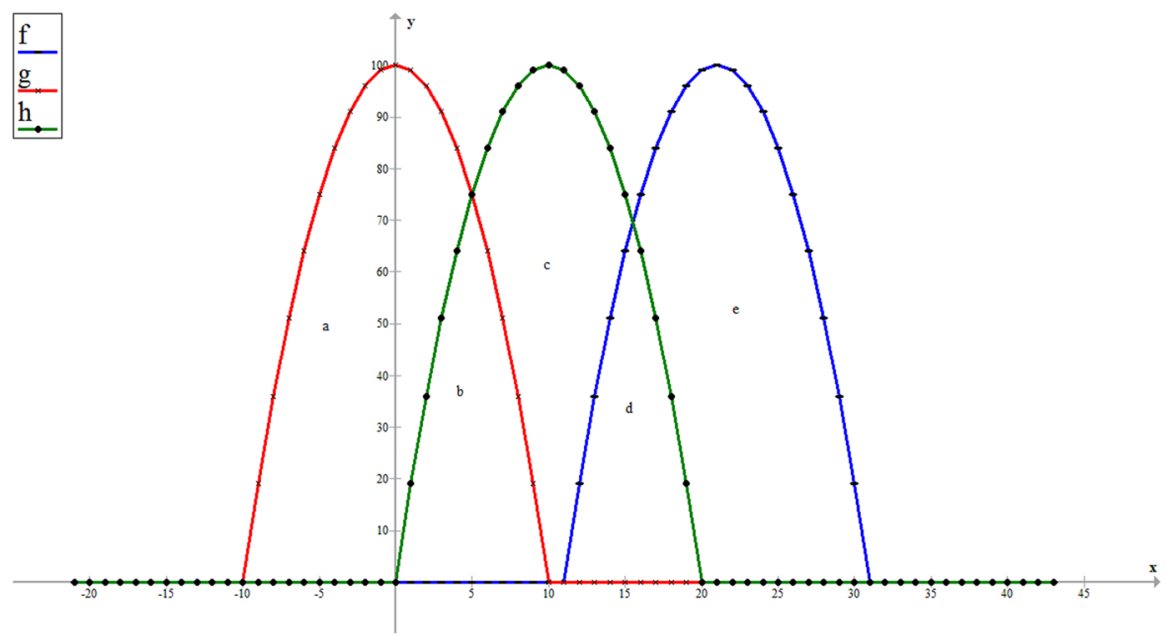

Figure 4. $A(f) \bigcap A(h) \neq \emptyset, A(g) \bigcap A(h) \neq \emptyset$ and $A(f) \bigcap A(g)=\emptyset$

a semi-terminating mapping on $\mathfrak{F}$, there exist $g, g^{*} \in \mathfrak{F}$ such that, $U$ is both $g$-order preserving and $\left[g, g^{*}\right]$-order preserving on $\mathfrak{F}$, and the below conditions hold:

$$
\begin{aligned}
& U^{i} g \preceq_{p} g^{*}, \forall i \in \mathbb{N} \\
& g \preceq_{p} U g .
\end{aligned}
$$

Proof. The semi-terminating conditions (2.5) and (2.7) follow immediately from the facts that, $\preceq_{p}$ is reflexive, and the invariant probability density function being globally stable. Also, using $g^{*}=g=k$, where $k$ is the globally 


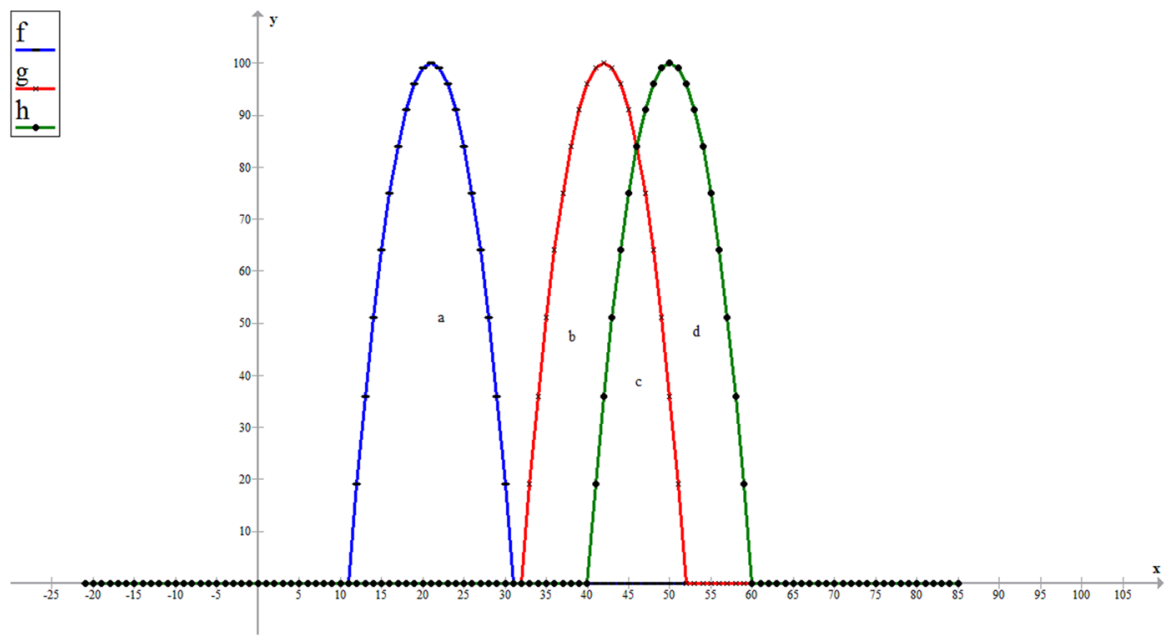

Figure 5. $A(f) \bigcap A(h)=\emptyset, A(g) \bigcap A(h) \neq \emptyset$ and $A(f) \bigcap A(g)=\emptyset$

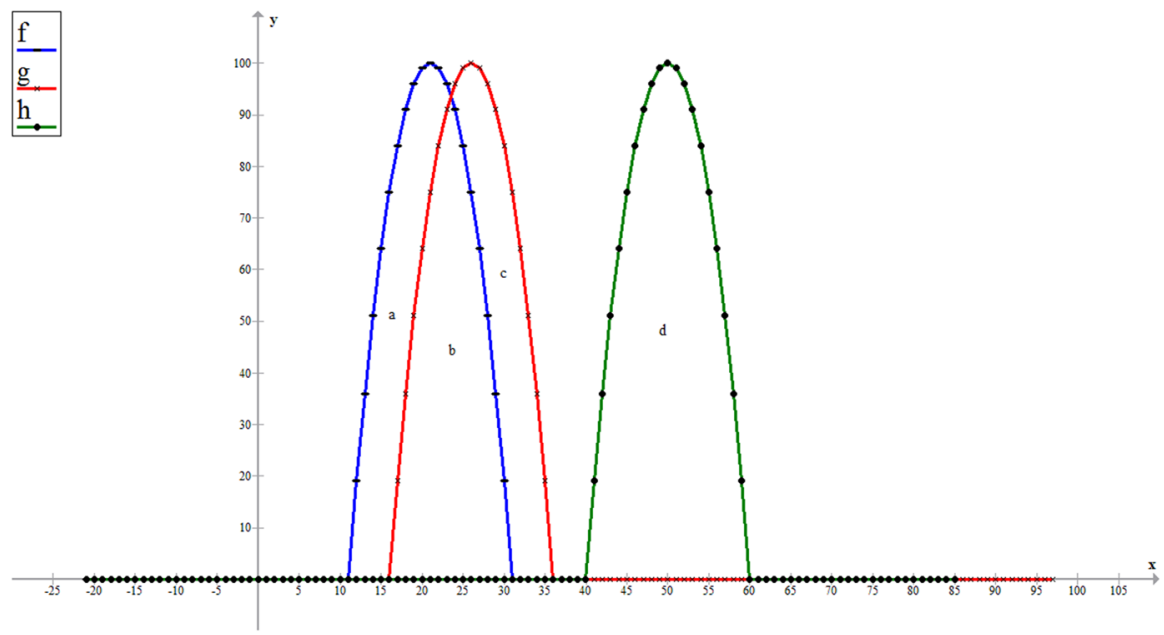

Figure 6. $A(f) \bigcap A(h)=\emptyset, A(g) \bigcap A(h)=\emptyset$ and $A(f) \bigcap A(g) \neq \emptyset$

stable invariant probability density function of $U$, then $U$ is both $g$-order preserving on $\mathfrak{F}$, and $\left[g, g^{*}\right]$-order preserving on $\mathfrak{F}$. Moreover, conditions (4.9) and (4.10) are trivially satisfied.

Conversely, the existence of an invariant probability density function follows immediately from Theorem 4.1. The uniqueness, and global stability of the invariant probability density function (fixed point) follow from the respective similar proves in Lemma 3.3.

The existence of $g^{*}$, satisfying (4.10) in Theorem 4.3 is by assuming $\left\{U^{i} f\right\}$ is tight; as provided by (4.9) and (4.10), see $[5,6]$. 

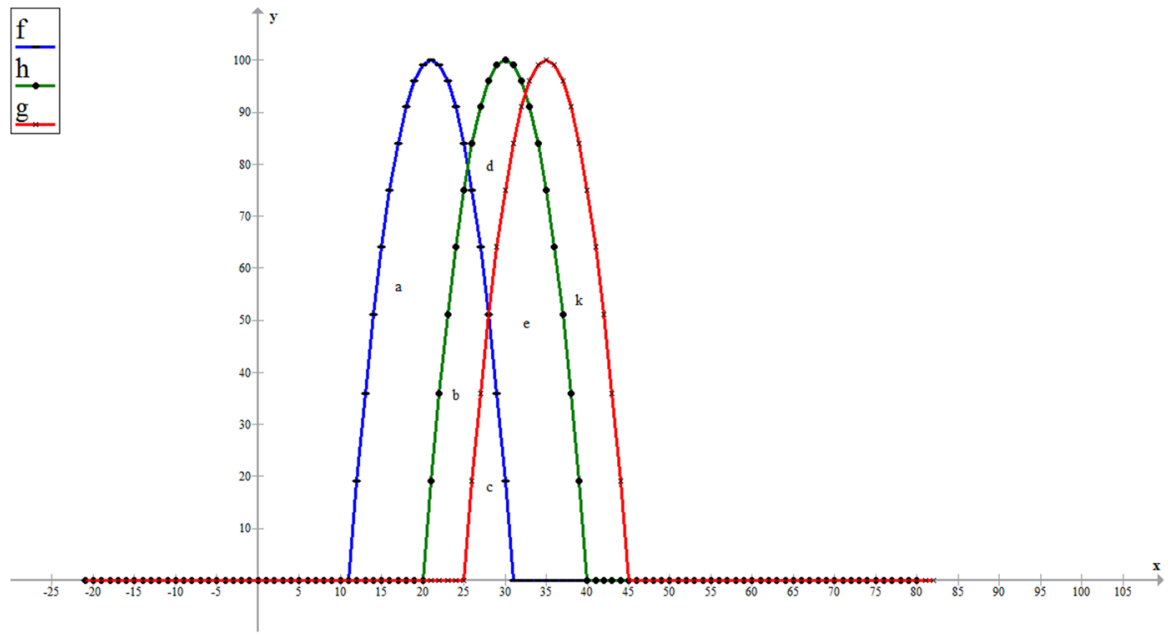

Figure 7. $A(f) \bigcap A(h) \neq \emptyset, A(g) \bigcap A(h) \neq \emptyset$ and $A(f) \bigcap A(g) \neq \emptyset$

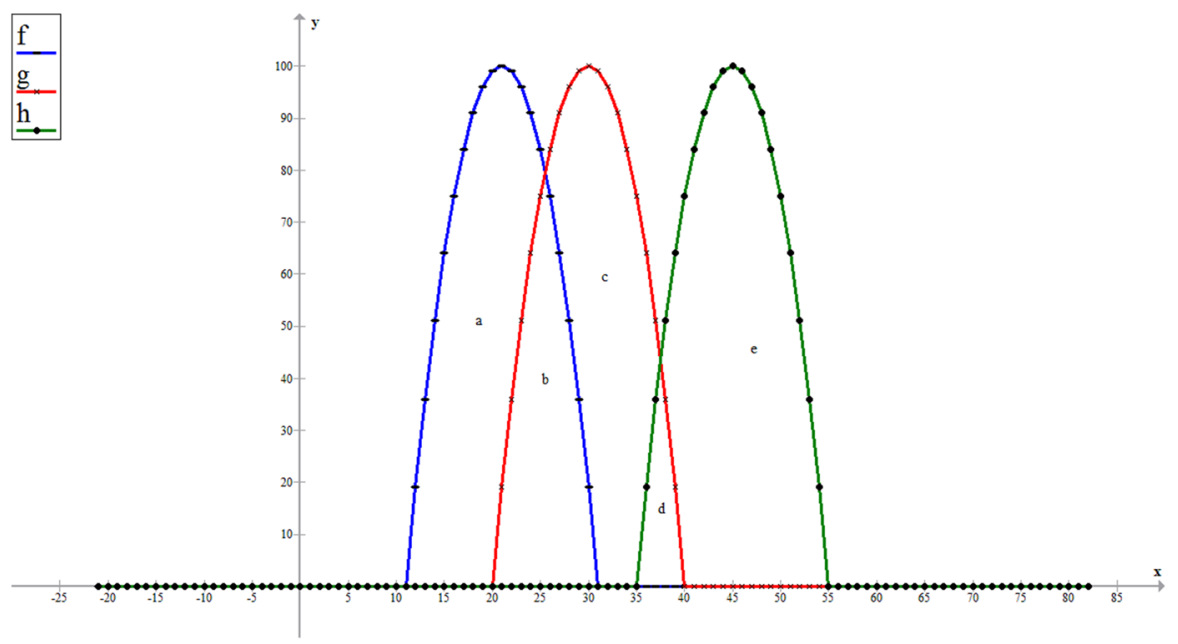

Figure 8. $A(f) \bigcap A(h)=\emptyset, A(g) \bigcap A(h) \neq \emptyset$ and $A(f) \bigcap A(g) \neq \emptyset$

\section{Application on quantum operations}

Consider the Bloch sphere below and denote it by Q (Fig. 9).

The states of a single bit two-level $(|0\rangle,|1\rangle)$ quantum bit (qubit) are described by the Bloch sphere below with $0 \leq \theta \leq \pi, 0 \leq \varphi \leq 2 \pi$; qubit is just a quantum system. Consider the phase flip transformation $(\mathcal{E})$ of the Bloch sphere defined by 


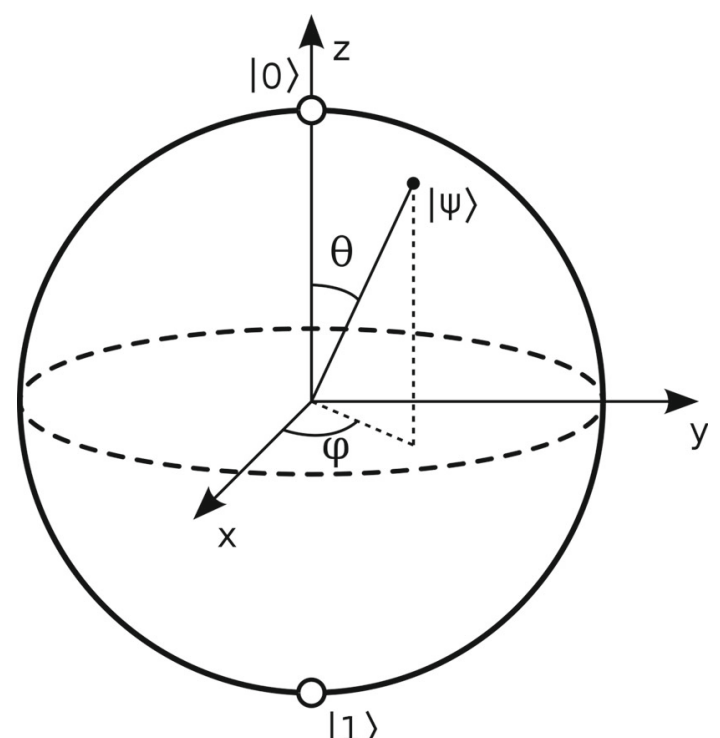

Figure 9. Cross section of a Bloch sphere

$$
\begin{aligned}
\mathcal{E}(\rho) & =\sum_{i=0}^{1} T_{i} \rho T_{i}^{\dagger}, \\
T_{0} & =\left[\begin{array}{cc}
\sqrt{\frac{1+\cos \theta}{2}} & \text { for } \\
0 & \sqrt{\frac{1+\cos \theta}{2}}
\end{array}\right], T_{1}=\left[\begin{array}{cc}
\sqrt{1-\frac{1+\cos \theta}{2}} & 0 \\
0 & -\sqrt{1-\frac{1+\cos \theta}{2}}
\end{array}\right],
\end{aligned}
$$

where $\mathcal{E}: Q \longrightarrow D \subseteq Q$ is a quantum operation, $\rho$ are the quantum states on or in the Bloch sphere. Define the order relation $\preceq$ on $Q$ by $\rho \preceq \gamma$ implies $\left|\rho_{a}+\rho_{b}+\rho_{c}\right| \geq\left|\gamma_{a}+\gamma_{b}+\gamma_{c}\right|$ for

$$
\begin{aligned}
\rho & =\left[\begin{array}{cc}
1+\rho_{a} & \rho_{b}-i \rho_{c} \\
\rho_{b}+i \rho_{c} & 1-\rho_{a}
\end{array}\right], \\
\gamma & =\left[\begin{array}{cc}
1+\gamma_{a} & \gamma_{b}-i \gamma_{c} \\
\gamma_{b}+i \gamma_{c} & 1-\gamma_{a}
\end{array}\right], \quad \text { for } \rho_{a}, \rho_{b}, \rho_{c}, \gamma_{a}, \gamma_{b}, \gamma_{c} \in[-1,1] .
\end{aligned}
$$

Also, define the functional $p: X \times X \longrightarrow(0,+\infty)$ by

$$
p(\rho, \gamma)=e^{\left|\rho_{a}-\gamma_{a}\right|+\left|\rho_{b}-\gamma_{b}\right|+\left|\rho_{c}-\gamma_{c}\right|} .
$$

But, each quantum state $\rho$ has a spherical representation as a Bloch vector $\bar{\rho}=\left[\rho_{b}, \rho_{c}, \rho_{a}\right]$ such that $\|\bar{\rho}\| \leq 1$. So in Theorem 3.1, if $U=\mathcal{E}, X=Q$; where $Q$ here represents the Bloch Sphere in Fig. 10. $s=\left[\frac{1}{m}, \frac{1}{m}, \frac{1}{m}\right]$ and $t=\left[0,0, \frac{1}{m}\right]$ for $\frac{1}{m} \in[-1,1]$. Then, Assumptions 3.1-3.4 are satisfied, all conditions of the theorem are also satisfied; hence, $\left\{\left[0,0, \frac{1}{m}\right]: \frac{1}{m} \in[-1,1]\right\}$ is the fixed point set of $\mathcal{E}$ (Fig. 11). 


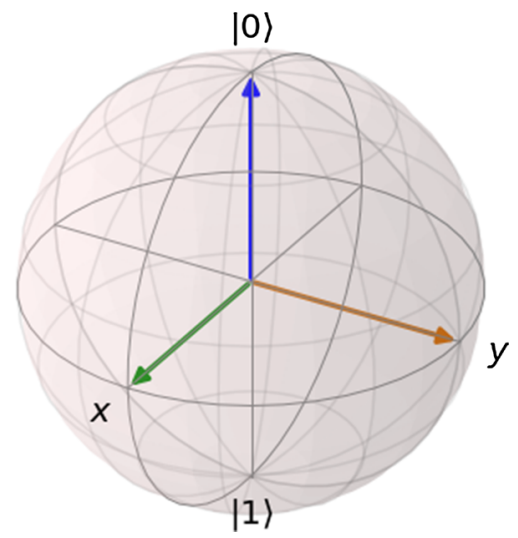

Figure 10. Before phase flip operation $(\mathcal{E})$

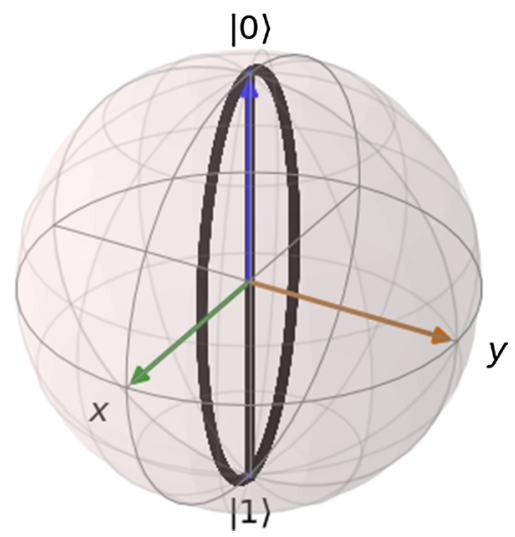

FiguRE 11. After phase flip operation $(\mathcal{E})$

In Fig. 11, the black ellipse in the Block sphere shows a cross section of an ellipsoid that is formed due to the effect of $T_{0}$ and $T_{1}$ on the states of the qubit, with $\frac{1+\cos \theta}{2}=0.44$. Furthermore, the black straight line in the Bloch sphere (Fig. 11) joining $(0,0,1)$ and $(0,0,-1)$ through the origin $(0,0,0)$ is the representation of the set of the fixed states of the qubit under the transformation $\mathcal{E}(5.1)$.

\section{Conclusion}

The result(s) in this paper,

1. extend(s) the work of Kamihigashi and Stachurski [5] from a metric space to a partial metric space.

2. improve(s) the work of Hassen [25] by establishing bi-conditional (iff) theorems, i.e., Theorems 4.2 and 4.3 compared to his Theorem 2.1. 
3. improve(s) the work of Altun and Erduran [26]. We provide more simpler prove, and do not use any continuity property on U if our Theorem 3.1 is compared with their Hassen [25] Theorem 2.1.

4. improve(s) Matthews's [7] Theorem 5.3; as our Theorem 3.1 requires no completeness property and is bi-conditional.

5. Fixed point sets of a quantum operation are used in information preserving structures(IPS); the fixed point set of $\mathcal{E}$ is isometric to some IPS.

Remark. The mapping $U$ we used is assumed to be nonlinear. As no linearity property was utilized. However, it can also work for linear cases like Markov chain. Finally, the idea of connecting the definition of $p$ on $\mathfrak{F}$ with the area of probability density functions may be extended to volumes in higher dimensions $\left(\mathbb{R}^{n}\right)$.

\section{Acknowledgements}

The authors acknowledge the financial support provided by King Mongkut's University of Technology Thonburi, through the "KMUTT 55th Anniversary Commemorative Fund". Umar Yusuf Batsari was supported by the Petchra Pra Jom Klao Doctoral Academic Scholarship for Ph.D. Program at KMUTT. Moreover, the coauthor was supported by Theoretical and Computational Science (TaCS) Center, under Computational and Applied Science for Smart Innovation research Cluster (CLASSIC), Faculty of Science, KMUTT.

\section{Compliance with ethical standards \\ Conflict of interest The authors have no competing interest.}

Author contributions The authors contributed equally, read and accepted the final version of the manuscript.

Open Access. This article is distributed under the terms of the Creative Commons Attribution 4.0 International License (http://creativecommons.org/licenses/by/4. 0/), which permits unrestricted use, distribution, and reproduction in any medium, provided you give appropriate credit to the original author(s) and the source, provide a link to the Creative Commons license, and indicate if changes were made.

Publisher's Note Springer Nature remains neutral with regard to jurisdictional claims in published maps and institutional affiliations.

Funding This research is supported through the research Grants of KMUTT and Petchra Pra Jom Klao Doctoral Academic Scholarship for Ph.D.

\section{References}

[1] Granas, A., Dugundji, J.: Fixed Point Theory. Springer, New York (2003)

[2] Stokey, N.L., Lucas Jr., R.E., Prescott, E.C.: Recursive Methods in Economic Dynamics. Harvard University Press, Massachusetts (1989) 
[3] Amanda, G.T.: Convegence of markov processes near a saddle fixed point. Ann. Probab. 35(3), 1141-1171 (2007)

[4] Heikkilä, S.: Fixed point results and their applications to markov processes. Fixed Point Theory Appl. 2005(3), 307-320 (2005)

[5] Kamihigashi, T., Stachurski, J.: Simple fixed point results for order-preserving self-maps and applications to nonlinear markov operators. Fixed Point Theory Appl. 2013, 351 (2013). https://doi.org/10.1186/1687-1812-2013-351

[6] Kamihigashi, T., Stachurski, J.: Stochastic stability in monotone economies. Theor. Econ. 9, 383-407 (2014)

[7] Matthews, S.G.: Partial metric topology. In: Proceedings of the 8th Summer Conference on General Topology and Applications. Annals of the New York Academy of Sciences, vol. 778, pp. 183-197 (1994)

[8] Onsod, W., Kumam, P., Cho, Y.J.: Fixed points of $\alpha-\Theta-$ geraghty type and $\Theta$-geraghty graphic type contractions. Appl. Gen. Topol. 18(1), 153-171 (2017). https://doi.org/10.4995/agt.2017.6694

[9] Tarski, A.: A lattice-theoretical fixed point theorem and its applications. Pac. J. Math. 5(2), 285-309 (1955)

[10] Agarwal, R., El-Gebeily, M.A., O'Regan, D.: Generalized contractions in partially ordered metric spaces. Appl. Anal. 87, 109-116 (2008)

[11] Bargetz, C., Dymond, M., Reich, S.: Porosity results for sets of strict contractions on geodesic metric spaces. Topol. Methods Nonlinear Anal. 50, 89-124 (2017)

[12] Aliprantis, C.D., Border, K.: Infinite Dimensional Analysis: A Hitchhikers Guide, 3rd edn. Springer, Berlin (2006)

[13] Goebel, K., Reich, S.: Uniform Convexity, Hyperbolic Geometry, and Nonexpansive Mappings. Mercel Dekker, New York (1984)

[14] Penot, J.P.: A fixed-point theorem for asymptotically contractive mappings. Proc. Am. Math. Soc. 131, 2371-2377 (2003)

[15] Reich, S., Shoikhet, D.: Nonlinear Semigroups, Fixed Points, and Geometry of Domains in Banach Spaces. Imperial College Press, London (2005)

[16] Shukla, S., Altun, I., Sen, R.: Fixed point theorems and assymptotically regular mappings in partial metric spaces. Comput. Math. 2013 (2013). https://doi. org/10.1155/2013/602579

[17] Matthews, S.G: Partial metric spaces. In: 8th British Colloquium for Theoretical Computer Science. Research Report 212, Dept. of Computer Science, University of Warwick (1992)

[18] Heckmann, R.: Approximation of metric spaces by partial metric spaces. Appl. Categ. Struct. 7(1-2), 71-83 (1999)

[19] Oltra, S., Valero, O.: Banach's fixed point theorem for partial metric spaces. Rend. Istit. Mat. Univ. Trieste XXXVI, 17-26 (2004)

[20] Batsari, U.Y., Kumam, P: A globally stable fixed point in an ordered partial metric space. In: Anh, L., Dong, L., Kreinovich, V., Thach, N. (eds.) Econometrics for Financial Applications. ECONVN 2018. Studies in Computational Intelligence, vol. 760, pp. 360-368. Springer International Publishing AG, Cham (2018). https://doi.org/10.1007/978-3-319-73150-6_29

[21] Knaster, B.: Un théorème sur les fonctions d'ensembles. Ann. Soc. Polon. Math. 6, 133-134 (1927) 
[22] Kamihigashi, T., Stachurski, J.: An order-theoretic mixing condition for monotone markov chains. Stat. Probab. Lett. 82, 262-267 (2012)

[23] Nieto, J., Rodríguez-López, R.: Existence and uniqueness of fixed point in partially ordered sets and applications to ordinary differential equations. Acta Math. Sin. Engl. Ser. 23, 2205-2212 (2007)

[24] Reem, D., Reich, S.: Zone and double zone diagrams in abstract spaces. Colloq. Math. 115, 129-145 (2009)

[25] Hassen, A.: Fixed point theorems for generalized weakly contractive condition in ordered partial metric spaces. J. Nonlinear Anal. Optim. 2(2), 269-284 (2011)

[26] Stephen, W.: General Topology, Dover edn. Dover Publication Inc, New York (2004)

Umar Yusuf Batsari and Poom Kumam

KMUTTFixed Point Research Laboratory, Room SCL 802 Fixed Point Laboratory, Science Laboratory Building, Department of Mathematics, Faculty of Science King Mongkut's University of Technology Thonburi (KMUTT)

126 Pracha-Uthit Road, Bang Mod, Thung Khru

Bangkok 10140

Thailand

e-mail: uyub2k@yahoo.com

Poom Kumam

e-mail: poom.kum@kmutt.ac.th

Umar Yusuf Batsari, Poom Kumam and Sompong Dhompongsa

KMUTTFixed Point Theory and Applications Research Group, Theoretical and Computational Science Center(TaCS), Science Laboratory Building

King Mongkut's University of Technology Thonburi (KMUTT)

126 Pracha-Uthit Road, Bang Mod, Thung Khru

Bangkok 10140

Thailand

e-mail: s.dhompongsa@gmail.com

Umar Yusuf Batsari

Department of Mathematics and Statistics, College of Science and Technology

Hassan Usman Katsina Polytechnic

Dutsin-Ma road, P.M.B. 2052

Katsina, Katsina State

Nigeria 